\title{
Can We Build Artificial Stem Cell Compartments?
}

\author{
Carlos E. Semino* \\ Center for Biological Engineering and Biotechnology Process Engineering Center, Building 56-354, \\ Massachusetts Institute of Technology, Cambridge, MA 02139, USA
}

Received 18 April 2002; revised 15 May 2002; accepted 21 May 2002

\begin{abstract}
Animals carry stem cells throughout their entire life, from embryogenesis to senescence. Their function during development and adulthood consists basically of forming and sustaining functional tissues while maintaining a small self-renewing population. They reside in a complex three-dimensional environment consisting of other nearby cells extracellular matrix components, endogenous or exogenous soluble factors, and physical, structural, or mechanical properties of the tissues they inhabit. Can we artificially recreate tissue development such that stem cells can both self-renew and be instructed to mature properly? The main factors required to regulate the maintenance and differentiation of some types of stem cells are known. In addition, new bioengineered synthetic materials that mimic extracellular matrix components can be used as initial scaffolding for building stem cell microenvironments.
\end{abstract}

\section{INTRODUCTION}

Embryonic and adult stem cells share common characteristics but differ in certain intrinsic properties. Both have the capacity to differentiate into at least one functional cell type which defines them as uni-, multi-, or pluripotential. Instead, they differ in intrinsic cell division kinetics. For instance, during early embryonic development, the cellular environment constantly changes, and the maintenance of pluripotentiality is short and transitory. Embryonic stem cells possess high expansive capacity in order to increase rapidly the amount of pluripotent cells able to generate all different tissues of the embryo. On the other hand, adult stem cell behavior and environment are paradoxically opposite. Adult stem cells stay as unchanged as possible in adult tissue niches while producing progeny at lower dividing capacity. In order to achieve these two distinct mechanisms of cell maintenance and function, tissues regulate intrinsic and extrinsic factors, creating niches. Here, I review some of the main components present in embryonic and two adult stem cells, bone marrow and the skin microenvironments as well as propose the use of new synthetic scaffolds to artificially recreate stem cell niches in vitro.

\section{STEM CELLS AND NEW BIOCOMPATIBLE MATERIALS}

How can we recreate in vitro niches for embryonic and adult stem cells? Due to knowledge of their natural environments, we can isolate them and maintain them as undifferentiated as possible. Although there is a considerable amount of knowledge of the main factors required for stem cell maintenance and differentiation in vitro, many of the pieces are still missing. If we want to use stem cells to obtain highly specialized and functional bioengineered tissues, we need to work with developmental paradigms where we use artificial materials that mimic stem cell compartments. If stem cells self-renew in such compartments, we have much better chances in instructing them for proper differentiation. How can we prepare threedimensional environments that will provide an initial transient scaffold for stem cell homing in vitro? The main initial components we need are biocompatible, defined, and synthetic three-dimensional scaffold materials such as bioceramics, microfiber scale polymers, or nanofiber hydrogels. These materials already exist: bioceramics such as hydroxyapatite (HA) [1]; the polylactic and polyglycolic acids (PLA and PGA) [2]; and self-assembling peptide hydrogels [3]. Why are micro- or nanoscale fibers important? They provide the stem cells with an initial threedimensional (3D) scaffold. In the first case, HA has sufficient rigidity to be used as artificial bone matrix. PLA or PGA polymers provide less rigid structures of medium mechanical strength with $50-100 \mu \mathrm{m}$ pore size to which cells adhere and grow on a pseudo 3D environment because their pore dimensions are in the same order of magnitude of an average cell size. On the other hand, peptide hydrogels have poor mechanical strength but their pore size is in between $50-100 \mathrm{~nm}$ (1,000 times smaller), in which cells experience a truly $3 \mathrm{D}$ environment. They are free to grow, migrate, contact other cells, change in shape, and expose membrane receptors in a proper way. The hydrogels are permeable to gases, metabolites, and macromolecules. Like the natural extracellular matrix, these hydrogels embed cells but do not entrap them. The advantage of having defined and artificial materials is immense. It provides an ideal opportunity to control conditions for stem cell maintenance, instruction, and differentiation. 
The peptide hydrogel provides an additional advantage. It mimics the extracellular matrix (ECM) with very low signaling capacity suggesting that membrane receptor ligands (ie, small molecules, growth factors, cytokines, extracellular matrix components or their protein domains) can be easily added to specifically decorate the environment as desired. The process can be easily industrialized since the synthesis is controllable (the hydrogel consists of short peptides, 12-16 residues) and can easily be scaled up. The advantage of HA, CPC, PLA, and PGA lies in their mechanical resistance. Eventually, materials can be used together where physical or mechanical factors can be considered instructive for stem cell differentiation.

\section{EARLY DEVELOPMENT: A CHANGING ENVIRONMENT}

\section{The inner cell mass (ICM) and the origin of embryonic stem cells}

In the mouse, cell division or cleavage starts 18 hours after fertilization producing an eight-cell embryo stage in which the cells are identical. During this stage, the embryo suffers from compaction so that all the cells adhere to each other. At the 32-cell stage, the first cell differentiation program is initiated; the cells on the periphery form the trophectoderm cell layer (TE), an epithelial monolayer of cells enclosing an internal group of cells, or the inner cell mass (ICM) (see Figure 1a). The external membrane of the TE contains a large amount of glycoproteins that play an important role in implantation and later on participate in the formation of the placenta. The ICM is the first population of embryonic stem cells (ESCs), but their existence is transitory since they will rapidly differentiate to form the tissues of the embryo and extraembryonic membranes, such as the allantois and the amnion. Embryonic stem cells isolated from the ICM have pluripotent capacity. After either transplantation or maintenance in certain culture conditions in vitro, they can give rise to derivative cells of each of the three primary germ layers-ectoderm, mesoderm, and endoderm-but not to an entire organism. In addition, an intrinsic characteristic of ESCs is their capacity for symmetric self-renewal and expansion. When isolated and cultured in proper conditions, they can be expanded to about $10^{10}$ undifferentiated cells.

\section{Cell adhesion and the extracellular matrix}

Cell adhesion plays an important role in keeping the ICM intact and is mediated mainly by cadherins and integrins. E-cadherin cell adhesion is regulated posttranslationally via protein kinase $\mathrm{C}$ and other signaling molecules. It coordinates cellular allocation and spatial organization of the ICM in the blastocyst [4]. $\beta 1$ integrins are required for normal morphogenesis and survival of the ICM. The interaction between the $\beta 1$ integrins and extracellular matrix components occurs via binding to laminin that is secreted by endodermal cells of the ICM [5]. In addition, proteoglycans, including embryoglycans composed of poly- $N$-acetyllactosamines, with molecular weight over $100 \mathrm{kd}$, are present on the surface of the compacted cells and play an important role in maintaining cell adhesion [6]. Embryoglycans carry a series of developmentally regulated oligosaccharide structures expressed in the embryonic ectoderm of early embryos, embryonic stem cells, and embryonic carcinoma cells [7]. The trisaccharide SSEA-1 (stage-specific embryonic antigen-1), with the structure $\operatorname{Gal}(\beta 1-4)[\operatorname{Fuc}(\alpha 1-$ $3)] \mathrm{GlcNac}$, mediates cell adhesion in pre-implanted embryos [8]. Moreover, the embryoglycan-carrying SSEA1 epitope might serve as a regulator in the signal transduction pathway of fibroblast growth factor-2 (FGF-2) and the high-affinity transmembrane receptor fibroblast growth factor-1 (FGFR-1) [9]. SSEA-1 acts as a recognition molecule for FGF-2, playing a role in ligand-receptor dimerization and regulating the mitotic effect of FGF-2 in embryonic stem cells [9].

\section{Oct-4 and LIF: prerequisites for ESCs pluripotentiality}

The transcription factor Oct-4 is essential for stem cells originated from ICM because it regulates the expression of downstream genes involved in maintaining ICM pluripotential capacity such as FGF-4. Oct-4 is expressed in all cells during the cleavage stage and becomes restricted to the ICM at the blastula stage [10]. In addition, the leukemia inhibitory factor (LIF) also controls the self-renewing capacity and undifferentiated state of ESCs in culture [11]. ESCs can be maintained indefinitely in culture with pluripotential capacity in the presence of LIF. LIF is expressed in the TE and secreted into the ICM. LIF operates through heterodimerization of two different classes of cytokine receptors that are expressed in ICM cells, the low-affinity LIF receptor (LIF-R), and the IL-6 signal transducer gp130 [12]. After LIF-induced dimerization, several tyrosine residues in the cytoplasmic domain of gp130 are phosphorylated by JAK kinases. The phosphorylated domain of gp130 interacts with the SH2 domain of the transcription factor STAT3, and, as a consequence, STAT3 is activated. The activation of STAT3 is sufficient for ESC self-renewal and maintenance in vitro [13]. Nevertheless, in vivo there is no requirement for LIF, gp130, or STAT3, indicating that the expansion of the epiblast is also under the control of unknown parallel signaling pathways which are not well known. This multilateral strategy demonstrates that the developmental program relies on alternative complementary pathways.

\section{ADULTHOOD: THE CHALLENGE FOR MAINTENANCE}

\section{Fighting the changes}

During animal development, cells differentiate and give rise to all tissues of the organism. Adult tissues maintain regenerative capacity based on certain minimal populations of stem cells that share similar properties with embryonic stem cells: multipotency and self-renewing capacity. For instance, adult tissues regulate cell mass and 


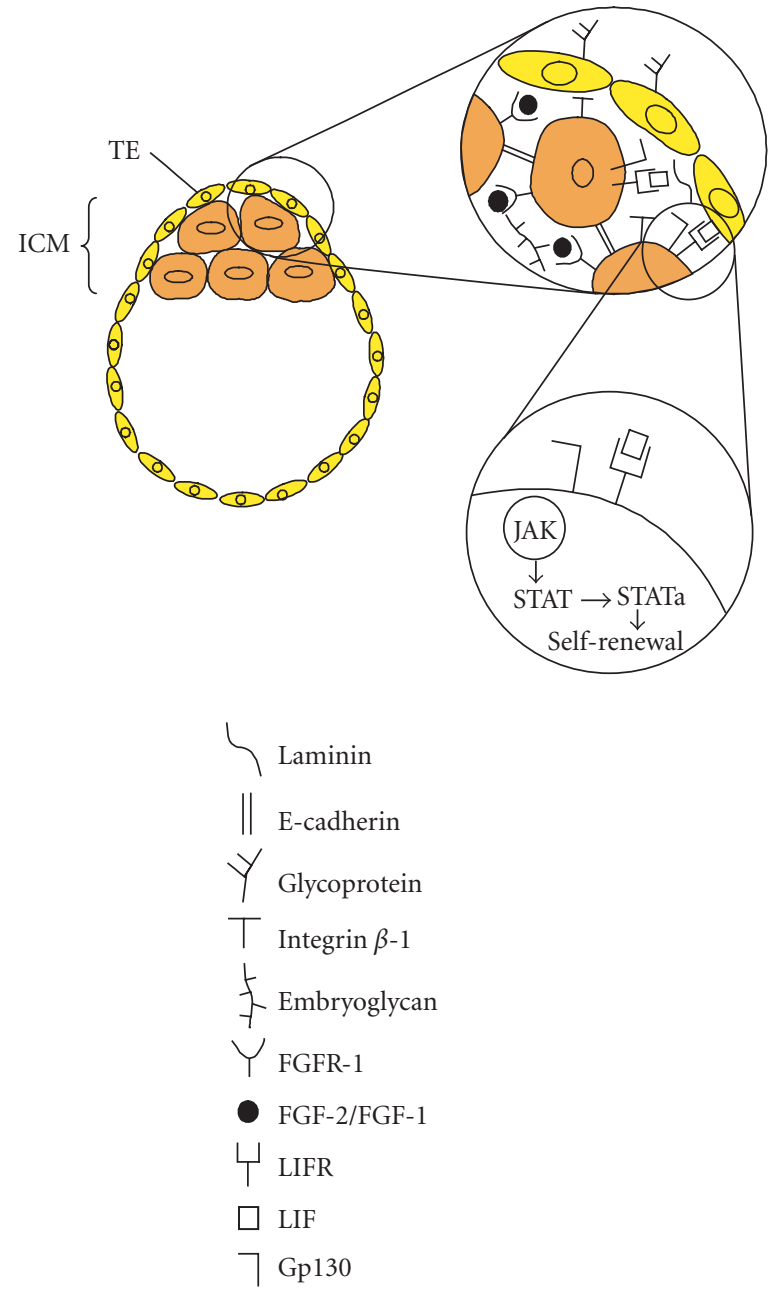

(a) Blastocyst
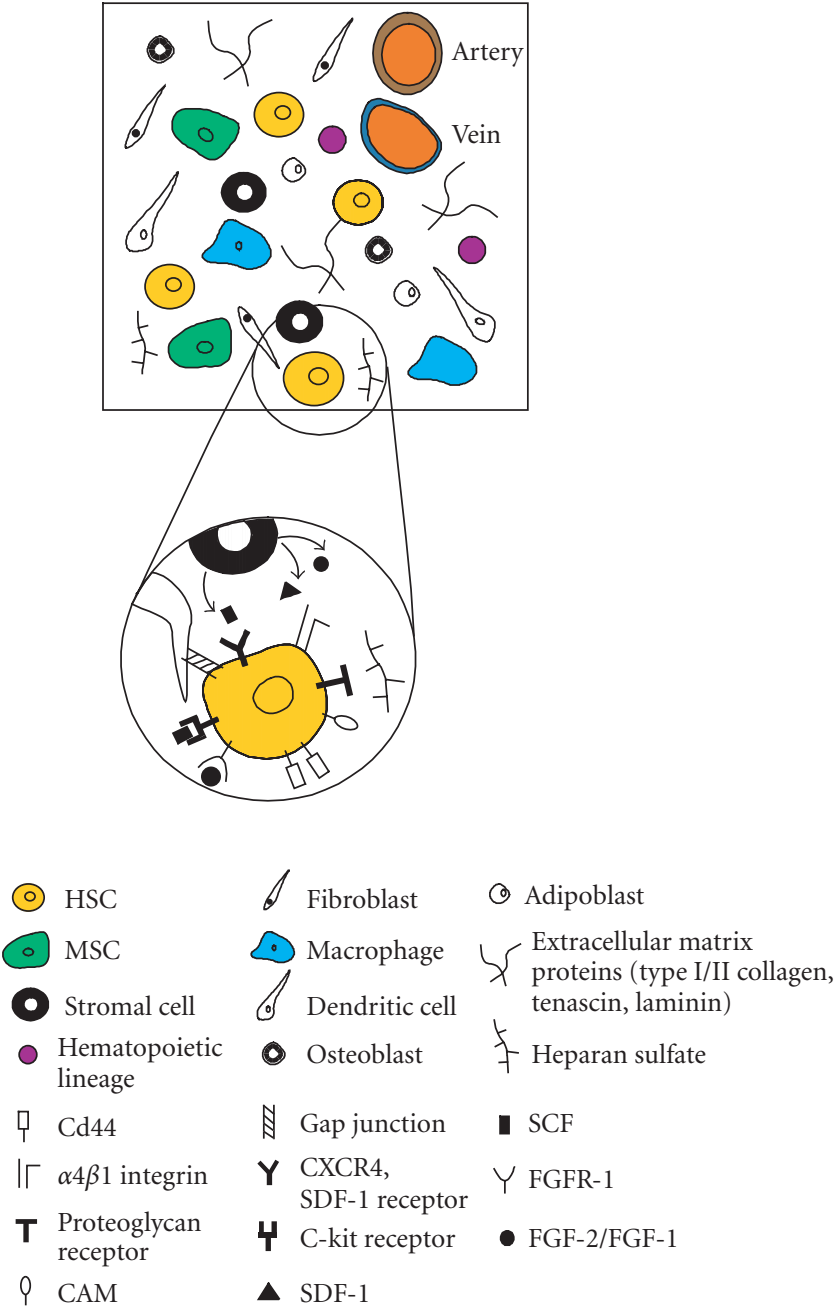

(b) Bone marrow

FIGURE 1. Schematic representation of the main components present in the blastocyst and bone marrow microenvironment. (a) Preimplanted embryo (blastocyst) composed by the inner cell mass (ICM) and the trophectoderm cell layer (TE). Abbreviations: FGF2/FGF, fibroblast growth factor 2 and 1; FGFR-1, fibroblast growth factor receptor-1; LIF, leukemia inhibitory factor; LIFR, leukemia inhibitory factor receptor; JAK, JAK kinase; STAT, transcription factor STAT; STATa, transcription factor STAT activated. (b) Adult bone marrow composed by HSCs, hematopoietic stem cells; MSCs, mesenchymal stem cells; and other cellular components such as adipocytes, stromal cells, hematopoietic lineage, macrophages, dendritic cells, osteoblasts, and adipoblasts. In addition, it is composed also by extracellular matrix proteins such as types I and II collagen, tenascin, laminin, and the proteoglycan heparan sulphate. Abbreviations: SDF-1, stroma-derived factor-1; SCF, stem cell factor; CAM, cell adhesion molecule.

shape by a mechanism involving cell expansion based, in part, on stem cell self-renewal through asymmetric cell division. This type of cell division produces two distinct cell daughters: a committed differentiated cell and another one identical to the original stem cell. In addition, adult tissues, unlike those in the early embryo, keep the microenvironment around the stem cells unchanged as long as possible. Adult stem cells divide slowly in tissues, and in general, the actively dividing cells are the "transitory cells" that are already committed to a tissue type. However, in certain tissue injuries, such as skin wounds, it is clear that the surrounding progenitor cells or transitory cells around the damaged tissue rapidly expand by changing their mitotic activity during healing. Intrinsic regulators, local chemical signals, and environmental factors govern this dramatic change in cell division and differentiation.

\section{The bone marrow microenvironment}

In the bone marrow, blood cells are constantly produced from hematopoietic stem cells (HSCs) by a mechanism of asymmetric cell divisions that generate a selfrenewing HSC and a progenitor cell. These progenitors ultimately generate all the cells in the hematopoietic lineage: red and white cells, eosinophils, mass cells, 
monocytes, neutrophils, $\mathrm{T}$ and $\mathrm{B}$ lymphocytes, and others [14] (see Figure 1b). But where do HSCs originate from? During embryogenesis, HSCs develop in the dorsal aorta, the yolk sack, and the lateral plate mesenchyme. This population of HSCs then migrates to the primitive liver, which becomes the hematopoietic organ of the fetus. Later, before birth, HSCs migrate to the bone marrow and stay there indefinitely. How do HSCs both regulate their progeny and maintain themselves? Part of the secret is based on stroma that is composed of many cells (mainly macrophages, fibroblasts, and adipocytes), and on an intrinsic property of stem cells self-renewal. Stromal cells are in intimate contact with HSCs, working as housekeeping cells by producing and maintaining the bone marrow microenvironment. These cells apparently interact physically with HSCs by gap junctions, intercellular channels that allow cytoplasmic exchange of small molecules between stromal cells and HSCs [15]. In addition, stromal cells create a three-dimensional environment by secreting ECM components, such as type I-IV collagens, tenascin, laminin, and heparan sulfate (HS) $[16,17,18]$. Stromal cells are positive for vimentin, muscle actin, CD10, and Stro-1 and negative for CD45. HSC adhesion to stroma involves a variety of membrane recognition molecules, such as $\alpha 4 \beta 1$ integrins which bind to fibronectin; the membrane receptor CD44 which interacts with the glucosaminoglycan hyaluronic acid (HA) [16]; and proteoglycan receptors acting in concert with cell adhesion molecules (CAMs), which recognize heparan sulfate [17]. The large number of CAMs expressed by the HSCs includes members of the sialomucin family such as CD34, members of the immonoglobulin family such as CD31 and CD50, and ligands for selectines. This last group belongs to a class of membrane receptors that especially recognize carbohydrate structures [18]. Integrin-mediated interactions of HSCs with ECM have multiple functions including regulation of proliferation and survival in the bone marrow and adhesion. In addition, stromal cells produce a cytokine, stroma-derived factor-1 (SDF-1), which is a chemoattractant for $\mathrm{CD}_{3} 4^{+}$cells. CD $34^{+}$hematopoietic progenitors express the SDF-1 receptor CXCR4, which stimulates transendothelial migration of these cells in presence of SDF-1 [19]. The soluble membrane-associated stem cell factor (SCF) participates in stem cell maintenance and adhesion by interacting with the c-kit receptor on the HSC membrane. Other stromal factors include FGF1 and FGF-2 which contribute (in addition to SDF-1) to HSC survival, homing, homeostasis, and proliferation [20].

The bone marrow microenvironment is even more complex. It contains not only HSCs and stroma but also another stem cell type, the mesenchymal stem cell (MSC); adherent macrophages; antigen-presenting cells, or dendritic cells; endothelial cells; and mesenchymal origin cells such as osteoblasts and adipoblasts. The MSCs selfperpetuate as undifferentiated cells and also undergo differentiation to produce all the mesenchymal tissues inside and outside the bone marrow, including their own marrow stroma, bone, cartilage, tendon, fat, and muscle [21]. The MSCs have some characteristic cell surface markers, such as cytokine receptors (IL-1R, IL-3R, IL-4R, IL-6R, IL-7R), extracellular matrix receptors (ICAM-I, ICAM2, VCAM-1, ALCAM), hyaluronate receptors, integrins $(\alpha 1, \alpha 2, \alpha 3, \alpha \mathrm{A}, \alpha \mathrm{V}, \beta 1, \beta 2, \beta 3, \beta 4)$, growth factor receptors (BFGFR, PDGFR), and other receptors (Thy-1, IFN $\gamma$ R, TGF $\beta$ R, TNFR) [22]. This basically indicates the complexity of the cellular and molecular interactions in which HSCs and MSCs are engaged. Interestingly, from the structural point of view, the bone marrow can be considered as a soft tissue surrounded by hard tissue residing in the internal part of the large bones. In other words, it is not exposed to strong mechanical loading in the way that cartilage, tendon, and muscle are. This could be one important factor in maintaining MSCs undifferentiated, and the exposure to mechanical forces in combination with specific microenvironment would dictate the differentiation into cartilage, tendon, or muscle tissues after MSCs colonize other tissues.

\section{Skin and epidermal stem cells}

The skin is of ectodermal origin, and is constantly being renewed at a high rate. It is organized in four main layers. The innermost layer, a thin layer of cells called the basal layer, is covered with a thick layer of cells, the spinous layer. Above these layers is the granular layer, which is covered with dead cells, or stratum corneum (our external skin). The basal layer undergoes active mitosis, constantly producing cells that migrate to the upper layers and terminally differentiate. The skin is a dynamic flow of cells from the inner basal layer to the surface. The epidermal stem cells, or high proliferative capacity keratinocytes, reside in the basal layer. Interestingly, the adherent properties of the epidermal stem cells and the high content of the extracellular matrix are what keep these cells attached to their microenvironment. They express surface integrins that adhere to collagen type IV ( $\alpha 2 \beta 1$ receptor) and to fibronectin ( $\alpha 5 \beta 1$ receptor), as well as low levels of the intercellular junction protein, E-cadherin [23]. This suggests that they interact strongly with the extracellular matrix and poorly with each other. In addition, epidermal stem cells contain a high level of $\beta$-catenin, suggesting that Wnt/Frizzled signaling is involved in the regulation of cell proliferation/differentiation of epidermal stem cells. Moreover, $\beta$-catenin has been shown to interact with E-cadherin, thus promoting cell adhesion. An excess of $\beta$-catenin will interact with other proteins such as Tcf, Groucho, SMAD4, CtBP, and CBP to form a transcription complex that interacts with DNA, regulating transcription of target genes such as CyclinD1 (cell cycle commitment) and c-MYC (exit from the stem cell compartment) [24, 25]. Exit of the stem cell compartment is produced by a decrease in the levels of integrins, thus reducing adhesion to the ECM. As a consequence, epidermal stem cells enter cell cycle arrest and terminal differentiation. 


\section{CONCLUSIONS}

In summary, some examples of essential biomaterials and stem cell niches have been described. In this final part, our intention is to illustrate to the readers to some examples of the combined use of two emerging disciplines, material science and stem cell technology. For instance, with the potential of building better stem cell environments, can we find conditions in which ESCs can be instructed in vitro to become an adult stem cell by controlling its microenvironment? By recreating developmental programs in designed 3D environments, ECSs can experience a rational sequence of factors (growth factors, extracellular matrix components, mechanic stimulus) required to mimic embryonic development. In a similar way, HSCs can be seeded in hydrogels loaded with purified extracellular matrix components mimicking bone marrow. The maintenance and expansion capacity of long-term HSCs can be challenged into these new compartments as well as their capacity to generate blood components. It will be extremely important to artificially regenerate blood or some of their main components in vitro. Can mechanical inputs be considered as a part of stem cell instructive factors? For instance, tubes of PGA mimicking arteries and veins can be built, and their microscale pores can be filled with hydrogel, clonally derived stem cells, microenvironment decorations, and flow frequencies applied. This will provide a similar tissue environment present during vertebrate circulatory system development. Similarly, for bone and cartilage, mesenchymal stem cells in the right microenvironment can be subjected to mechanical loading with a program that can recapitulate limb movement and mechanical strength during development. Finally, the skin area can also be explored in more detail. A basal layer-like structure can be easily obtained simply by applying collagens, laminins, and fibronectins onto a porous membrane where keratinocytes will attach, actively divide, and migrate to an upper layer of specifically decorated hydrogel. The flow of cells from the inner to the upper layers can be reproduced as in normal tissues with the hope to obtain better artificial skin. The use of new designed biocompatible material in combination with clonally derived stem cells is an emerging discipline with unlimited potential for future reparative medicine.

\section{ACKNOWLEDGMENTS}

I would like to specially thank my colleagues at MIT, Shuguang Zhang, Alan Grodzinsky, John Kisiday, Kim Hamad, Colette Shen, and Christine Ko, for many gratifying discussions that helped me to write this work. I would also like to thank the directors of the Center for Biological Engineering (CBE) and Biotechnology Process Engineering Center (BPEC) for their support.

\section{REFERENCES}

[1] Shikinami Y, Okuno M. Bioresorbable devices made of forged composites of hydroxyapatite (HA) parti- cles and poly-L-lactide (PLLA): Part I. Basic characteristics. Biomaterials. 1999;20(9):859-877.

[2] Perrin DE, English JP. Polyglycolite and polylactide. In: Domb AJ, Kost J, Wiseman DM, eds. Handbook of Biodegradable Polymers. New York: Harwood Academic Publishers; 1997:3-27.

[3] Holmes TC, de Lacalle S, Su X, Liu G, Rich A, Zhang S. Extensive neurite outgrowth and active synapse formation on self-assembling peptide scaffolds. Proc Natl Acad Sci USA. 2000;97(12):67286733.

[4] Fleming TP, Sheth B, Fesenko I. Cell adhesion in the preimplantation mammalian embryo and its role in trophectoderm differentiation and blastocyst morphogenesis. Front Biosci. 2001;6:D1000D1007.

[5] Stephens LE, Sutherland AE, Klimanskaya IV, et al. Deletion of beta 1 integrins in mice results in inner cell mass failure and peri-implantation lethality. Genes Dev. 1995;9(15):1883-1895.

[6] Wight TN, Heinegard DK, Hascall VC. Proteoglycans. Structure and function. In: Hay E, ed. Cell Biology of the Extracellular Matrix. New York: Plenum Press; 1991:45-78.

[7] Muramatsu T. Early embryogenesis. In: Fukuda M, ed. Cell Surface Carbohydrates and Cell Development. Boca Raton, Florida: CRC Press; 1992:239-256.

[8] Kojima N, Fenderson BA, Stroud MR, et al. Further studies on cell adhesion based on $\operatorname{Le}(\mathrm{x})-\mathrm{Le}(\mathrm{x})$ interaction, with new approaches: embryoglycan aggregation of F9 teratocarcinoma cells, and adhesion of various tumour cells based on $\mathrm{Le}(\mathrm{x})$ expression. Glycoconj J. 1994;11(3):238-248.

[9] Dvorák P, Hampl A, Jirmanová L, Pacholíková J, Kusakabe M. Embryoglycan ectodomains regulate biological activity of FGF-2 to embryonic stem cells. J Cell Sci. 1998;111(pt 19):2945-2952.

[10] Palmieri SL, Peter W, Hess H, Scholer HR. Oct4 transcription factor is differentially expressed in the mouse embryo during establishment of the first two extraembryonic cell lineages involved in implantation. Dev Biol. 1994;166(1):259267.

[11] Williams RL, Hilton DJ, Pease S, et al. Myeloid leukaemia inhibitory factor maintains the developmental potential of embryonic stem cells. Nature. 1988;336(6200):684-687.

[12] Gearing DP, Thut CJ, VandeBos T, et al. Leukemia inhibitory factor receptor is structurally related to the IL-6 signal transducer, gp130. EMBO J. 1991;10(10):2839-2848.

[13] Niwa H, Burdon T, Chambers I, Smith AG. Selfrenewal of pluripotent embryonic stem cells is mediated via activation of STAT3. Genes Dev. 1998;12(13):2048-2060.

[14] Weissman IL. Stem cells: units of development, units of regeneration, and units in evolution. Cell. 2000;100(1):157-168. 
[15] Montecino-Rodriguez E, Dorshkind K. Regulation of hematopoiesis by gap junction-mediated intercellular communication. J Leukoc Biol. 2001;70(3):341347.

[16] Clark BR, Keating A. Biology of bone marrow stroma. Ann N Y Acad Sci. 1995;770:70-78.

[17] Siczkowski M, Clarke D, Gordon MY. Binding of primitive hematopoietic progenitor cells to marrow stromal cells involves heparan sulfate. Blood. 1992;80(4):912-919.

[18] Whetton AD, Spooncer E. Role of cytokines and extracellular matrix in the regulation of haemopoietic stem cells. Curr Opin Cell Biol. 1998;10(6):721-726.

[19] Mohle R, Bautz F, Rafii S, Moore MA, Brugger W, Kanz L. The chemokine receptor CXCR-4 is expressed on $\mathrm{CD} 34+$ hematopoietic progenitors and leukemic cells and mediates transendothelial migration induced by stromal cell-derived factor-1. Blood. 1998;91(12):4523-4530.

[20] Whetton AD, Graham GJ. Homing and mobilization in the stem cell niche. Trends Cell Biol. 1999;9(6):233-238.

[21] Pittenger MF, Mackay AM, Beck SC, et al. Multilineage potential of adult human mesenchymal stem cells. Science 1999;284(5411):143-147.

[22] Pittenger MF, Marshak DR. Regenerative mesenchymal stem cells from adult bone marrow. In: Marshak DR, Gardner RL, Gottlieb D, eds. Stem Cells. Cold Spring Harbor, NY: Cold Spring Harbor Laboratory Press; 2001:349-373.

[23] Moles JP, Watt FM. The epidermal stem cell compartment: variation in expression levels of $\mathrm{E}$ cadherin and catenins within the basal layer of human epidermis. J Histochem Cytochem. 1997; 45(6):867-874.

[24] Tetsu O, McCormick F. Beta-catenin regulates expression of cyclin D1 in colon carcinoma cells. $\mathrm{Na}$ ture. 1999;398(6726):422-426.

[25] He TC, Sparks AB, Rago C, et al. Identification of c-MYC as a target of the APC pathway. Science. 1998;281(5382):1509-1512.

\footnotetext{
* E-mail: semino@mit.edu
} 

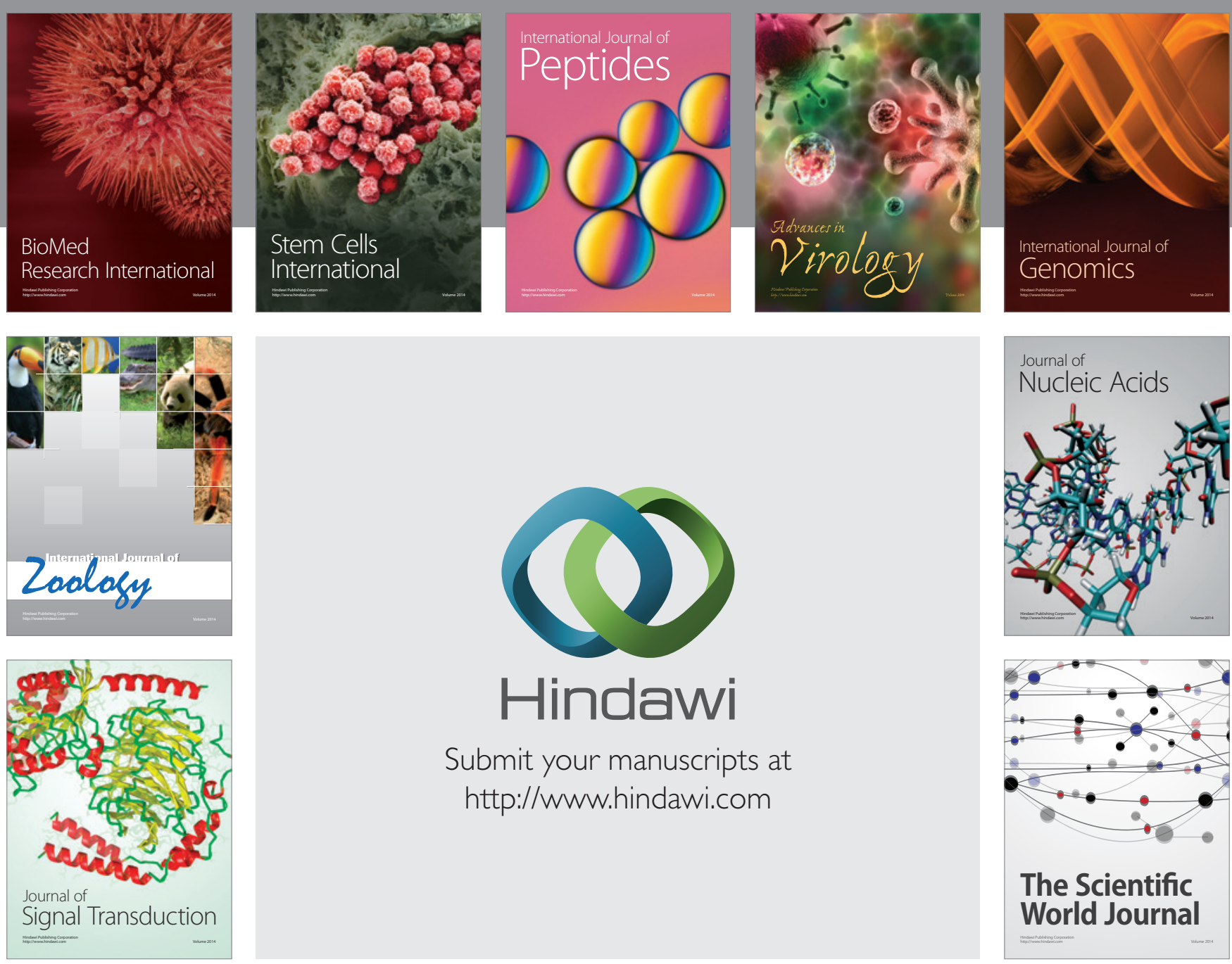

Submit your manuscripts at

http://www.hindawi.com
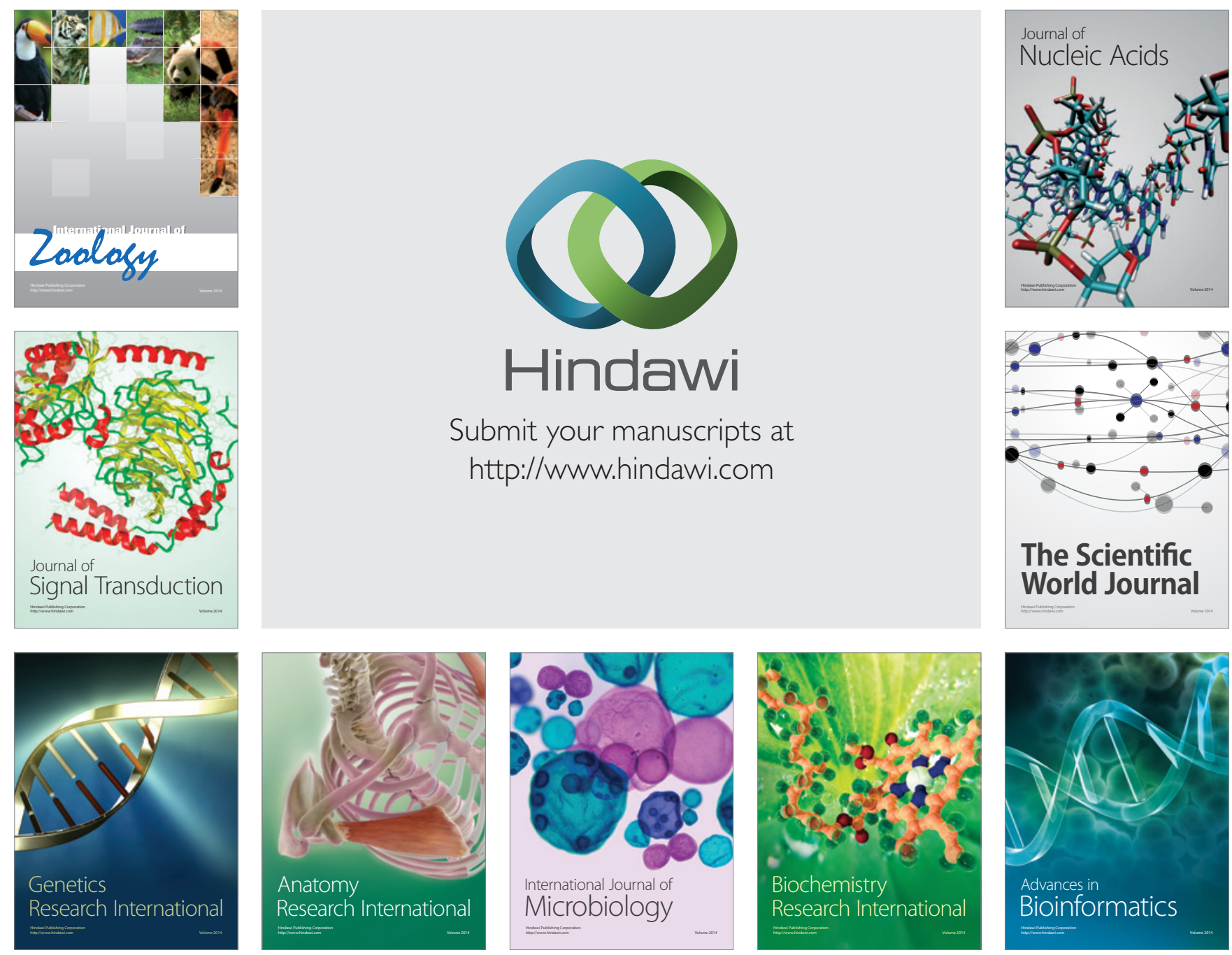

The Scientific World Journal
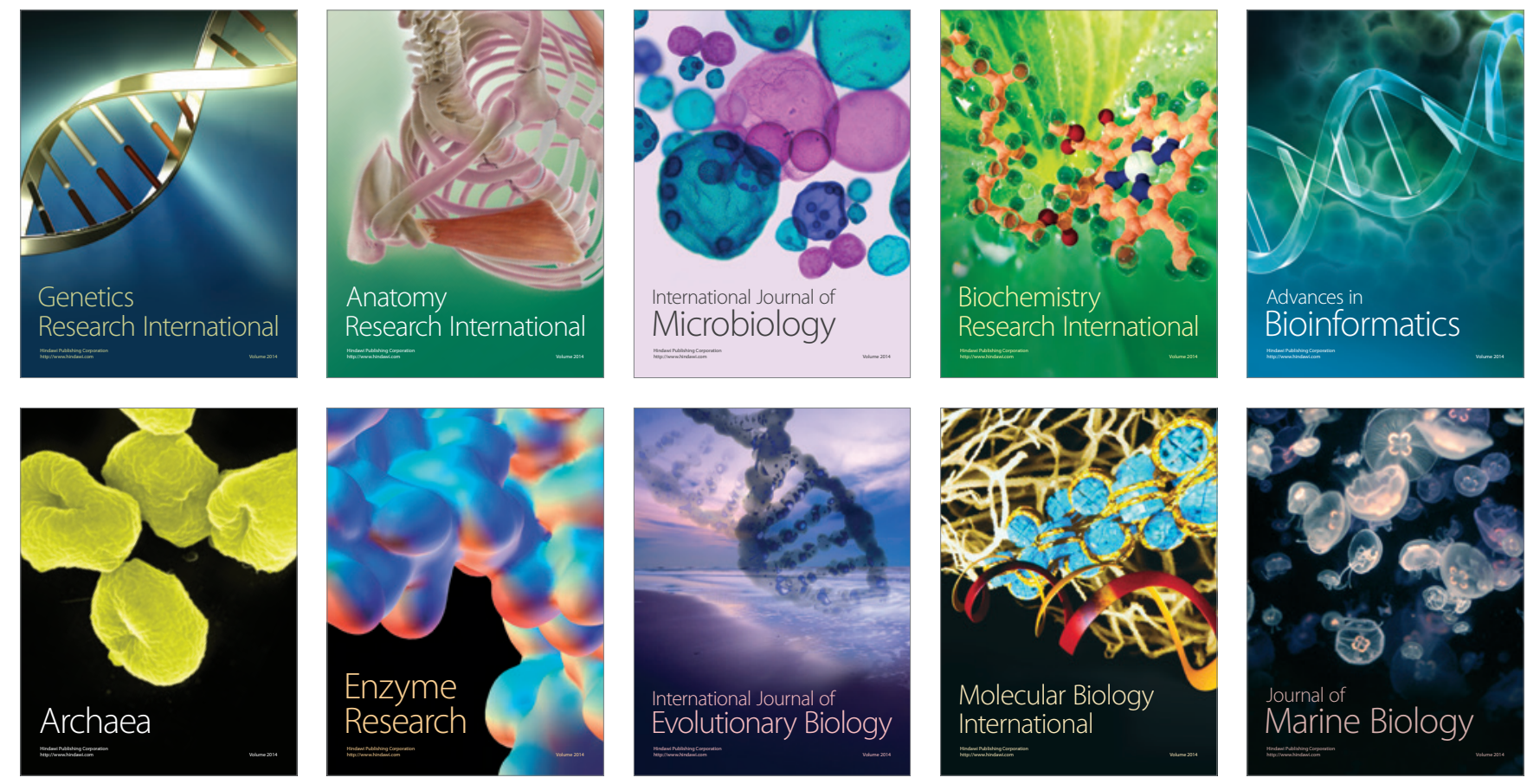